\title{
Invitation to the Cosmic Ray Extremely Distributed Observatory
}

Piotr Homola ${ }^{a, *}$ on behalf of the CREDO Collaboration

(a complete list of authors can be found at the end of the proceedings)

a Institute of Nuclear Physics Polish Academy of Sciences, ul. Radzikowskiego 152, 31-342 Kraków, Poland

E-mail: Piotr.Homola@ifj.edu.pl

Cosmic Ray Ensembles (CRE) are very large, yet not observed particle cascades initiated above the Earth atmosphere. Such cascades could be formed both within classical models (e.g. products of photon-photon interactions) and exotic scenarios (e.g. result of decay of Super Heavy Dark Matter particles and subsequent interactions). Some of CRE might have a significant spatial extent which could serve as a unique signature detectable with the existing cosmic ray infrastructure taken as a network of detectors. This signature would be composed of a number of air showers with parallel axes. An obvious, although yet not probed, CRE ,detection horizon” can be located somewhere between an air shower induced by an CRE composed of tightly collimated particles (preshower effect), and undetectable CRE composed of particles spread so widely that only one of them have a chance to reach Earth. Probing the CRE horizon with a global approach to the cosmic ray data, as proposed by the newly formed Cosmic Ray Extremely Distributed Observatory (CREDO), defines an extensive scientific program oriented on the search for physics manifestations at largest energies known, with potential impact on ultra-high energy astrophysics, the physics of fundamental particle interactions and cosmology. In this talk the current status and perspectives of CREDO will be summarized, with an open invitation for the colleagues interested in a global approach to cosmic ray studies, and in particular in observing and investigating multi-primary cosmic ray events such as CRE.

$37^{\text {th }}$ International Cosmic Ray Conference (ICRC 2021)

July 12 th - 23rd, 2021

Online - Berlin, Germany

\footnotetext{
*Presenter
} 


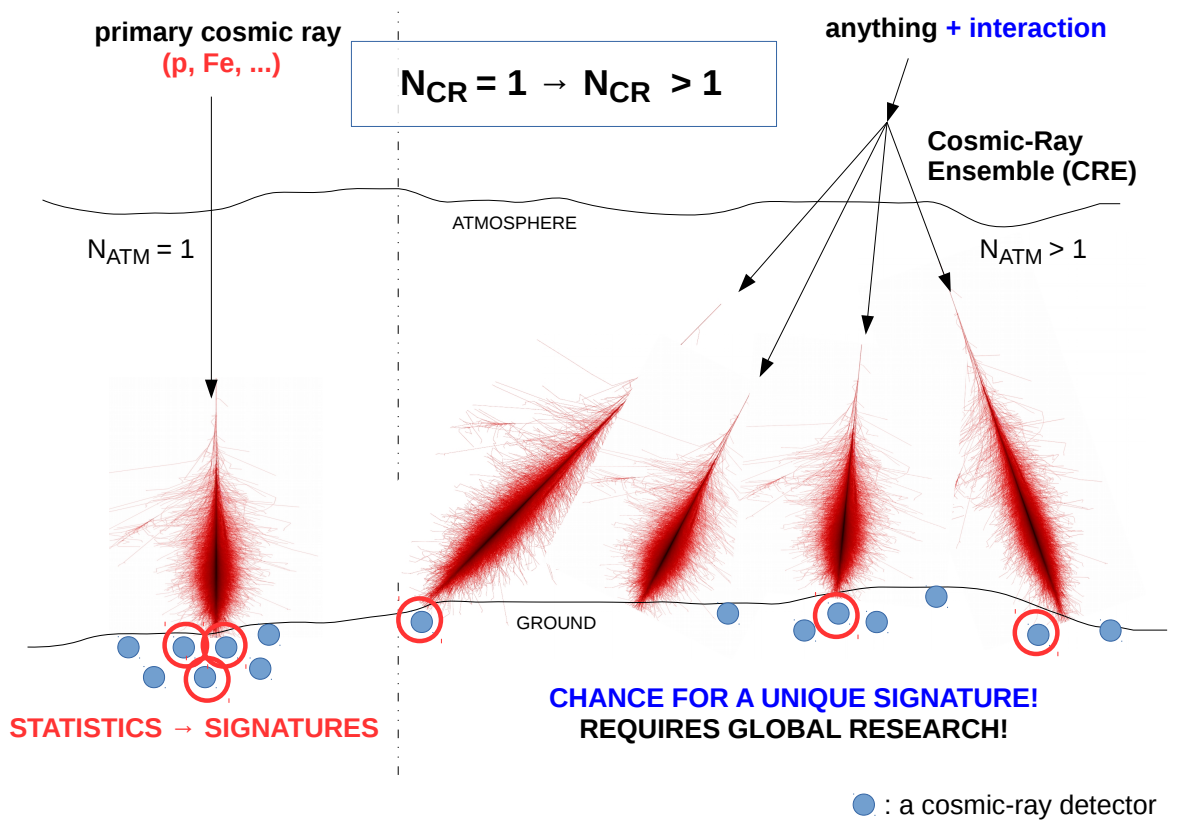

Figure 1: Cosmic Ray Ensembles: a novelty in cosmic ray research and in multi-messenger astroparticle physics. [1]

\section{Introduction}

High energy cosmic rays are expected to interact with radiation and matter on their way through the cosmos and give birth to the Cosmic Ray Ensembles (CRE) - the phenomena composed of at least two cosmic ray particles or photons, with a common primary interaction vertex or the same parent particle with correlated arrival directions and arrival times. It is therefore justified to ask under which circumstances and with which conditions CRE can reach the Earth and be at least partly detected with the available or possible infrastructure. The scientific novelty of the CRE-oriented research is schematically illustrated in Fig. 1, which demonstrates that the signatures of CRE might be spread over very large surfaces which might make them hardly detectable by existing detector systems operating individually. A relevant experimental strategy should be based on extensive simulations of the propagation of CRE particle distributions as predicted by theoretical models, including the simulations in intergalactic, interstellar and interplanetary environments, as within the Earth's atmosphere where high energy cosmic rays induce extensive air shower (EAS).

Since a general approach to studies related to CRE should not include restrictions concerning their origin and physical processes behind, one should then make no a priori assumptions about the properties of CRE or CRE parts that can be observed on Earth. In particular one can expect that physical processes occurring during the propagation of cosmic rays through the intergalactic and interstellar space give birth to very large cascades, both in terms of number of particles and their spatial extent. Also a significant temporal extent of arrival time distribution of CRE components can not be excluded a priori. Some of the examples of physical processes capable of generating CRE include both classical models (e.g. synchrotron radiation) and exotic scenarios (e.g. result of 
decay of Super Heavy Dark Matter particles and subsequent interactions). In case processes that initiate CRE occur at large distances from the observation point a significant spatial extent of the front formed by CRE components could serve as a unique signature detectable with the existing cosmic ray infrastructure taken as a network of detectors. This signature would be composed of a number of air showers with parallel axes. To observe such a multi-primary cosmic ray phenomenon it is then justified to apply a novel, simple though yet not probed strategy based on a global analysis of all available cosmic ray data aiming at reaching the sensitivity to extremely extended cosmic ray phenomena possibly invisible for individual detectors or observatories. Thus the search for CRE with a global approach to the cosmic ray data defines an extensive scientific program dedicated to observing and studying physics manifestations at largest energies known, with potential impact on ultra-high energy astrophysics, the physics of fundamental particle interactions and cosmology.

Such a program has been adopted and is being implemented by the Cosmic Ray Extremely Distributed Observatory (CREDO) Collaboration (see Ref. [1] and the topical references therin). To date (7.07.2021) the CREDO Memorandum of Understanding (MoU) has been signed by the representatives of 43 institutions from 19 countries on 5 continents (for the updated list of the institutional CREDO members please see https://credo.science/credo-institutional-members/). It is expected that the number of institutions engaged in the CREDO scientific program will grow rapidly to increase chances for new, valuable scientific results that can be possibly obtained only in a collective global effort of many researchers. It is important to emphasize that the invitation to CREDO has been and will be continuously open to all of the interested parties, including also the collaborations and institutions not only directly engaged with pure cosmic ray research or gamma astronomy, but also those which receive cosmic signal as a noise. Such a noise (e.g. muons in underground or underwater neutrino or Dark Matter experiments/observatories, radiation detected in CCD/CMOS pixel cameras used e.g. in classical astronomy, off-beam detections in sensors designed and working in experiments carried out in particle accelerators) can be a valuable scientific signal that feeds a global research strategy dedicated to CRE. It is also important to state here that CREDO is an open observatory which means that any institution or individual researcher can have an access to the data and software resources without formal joining the Collaboration the access is granted only after evaluating declared scientific motivations. Despite full openness of CREDO it is desirable that the interested institutions join under the MoU as this formal umbrella stimulates scientific and organizational synergies, and collaborative effort is known to outperform an individual approach to science, and especially to research that by definition has to carried out on a global scale.

Within this report we give an overview of the CREDO status and perspectives, as presented in the other ICRC 2021 articles and in the first several peer-reviewed articles that have been published within the last year.

\section{Theoretical encouragements}

The first step on the road map to observation and studies on CRE is a multi-scenario theoretical modeling and simulations concerning the possible sources and physics processes that might result in forming a cascade of particles collimated enough to be observed at least partly (at least two particles) at the Earth, or, thinking about future possibilities, within the reach of our techno sphere. 
The studies ongoing presently within the CREDO Collaboration include different possible scales of distances of the CRE sources or origins and several example physics processes, all highlighted all the ICRC 2021:

1. The preshower effect in the vicinity of the Earth $[2,3]$ : a new detection capability study dedicated to gamma telescopes;

2. The preshower effect in the vicinity of the Sun [4]: a demonstration of a new, characteristic footprint of UHE photons, "air shower walls", which is a very thin (few meters) and very elongated (from thousands to millions of kilometers) front of photons resulting from magnetic pair production undergone by an UHE photon passing nearby the Sun;

3. Synchrotron radiation of high energy electrons propagating in the galactic magnetic fields [5]: a new perspective of the possibility to investigate on multi primary cosmic ray events originated either nearby individual astrophysical sources, or from a scenario where a uniform distribution of emitting electrons is taken into account;

4. Acceleration of UHECR in the extreme environments near local (located within $100 \mathrm{Mpc}$ distance) supermassive black hole candidates, potentially leading to interactions capable of forming observable CRE [6]: a novel exploration of an ultra-efficient regime of the magnetic Penrose process, in which protons and ions are energized near SMBH by the ionization or decay of low-energy neutral particles, such as e.g. a hydrogen ionization or neutron beta-decay;

5. The models considered independently of the astrophysical location of their occurrence [7]: interactions of exotic super-heavy matter (including extra dimensions, Lorentz invariance violation, cosmic strings, dark matter particles or particles beyond the standard model etc.) and acceleration scenarios describing processes in which the particles are accelerated by a particular astrophysical object (shocks in relativistic plasma jets, unipolar induction mechanisms, second-order Fermi acceleration, energy transfer from black holes or compact stars etc.).

Although the above list is just an arbitrary selection of theoretical possibilities for CRE, it is already very encouraging. A particularly motivating result is that the CRE observation horizon extends over galactic distances and that the physics processes behind galactic CRE do not need to assume exotic models, as shown in Ref [5]. Another interesting and promising research direction is an attempt to observe CRE initiated by UHE photons passing nearby the Sun, as demonstrated in Ref [4]. The expected effect to be observed at the Earth, i.e. multi primary, very elongated air shower fronts, are UHE photon footprints that give chance for zero-background observations. Moreover, some of the lower energy photons located in the tales of such fronts might appear as arriving directly from the direction of the Sun. Such observations or their absence would be directly comparable with the results from gamma ray observatories to give new, independent constraints on UHE photon fluxes and the related physics, e.g. on super-heavy dark matter models . 


\section{The search for large scale cosmic ray correlations with the CREDO infrastructure}

The theoretical prospects for CRE observation directly motivate an effort towards designing and implementing observational strategies, as currently being performed by the CREDO Collaboration. While some types of CRE are detectable and probably also identifiable by individual big observatories like the Cherenkov Telescope Array (see the study in Ref. [2]), or even by smaller array (see the observational highlight in Ref. [8] and the references therin related to similar observations before), it is generally expected that CRE are widely spread collections of cosmic rays, with the correlation scale between the CRE constituents of the order of the size of the Earth, and such an expectation implies a global approach to the observational effort. Such an approach would adopt all the available cosmic ray signal, including both the professional infrastructure and the medium or small detectors, including private devices like smartphones. The use of small, portable, and affordable devices is motivated by a unique chance to cover very large areas of the globe with the radiation sensors, by a chance of engaging a large number of science enthusiasts of which some might undertake careers in science to help in the research on a higher level, and by relatively low investments required to spin off such a network. At present, the CREDO Collaboration focuses on developing strategies and solutions dedicated to supporting such a global network of small detectors, keeping the door an invitation open for the already well established collaborations that operate fully professional and sophisticated observatories or detectors - hoping for the synergy and optimum CRE-observation efficiency to arise from truly global and collective effort of all the interested individuals and institutions. The efforts towards supporting a global network of small and affordable cosmic ray detectors being undertaken by the CREDO Collaboration and presented at ICRC 2021 include:

1. Maintenance and development of applications that enable detection of radiation with mobile devices, and the related machine learning methods [9, 10]: the solutions for both Android and iOS are currently operational, and a considerable data base of candidate particle tracks recorded with CMOS sensors of mobile devices is being stored centrally, enabling in depth studies related to pattern recognition and classification of the record images are being developed, to form a sable basis for scientific analyses;

2. Analytical approach to identifying muons among the particle tracks recorded by mobile devices [11, 12]: the straight particle tracks observed by mobile devices correspond to passages of muons which has been shown by comparing a distribution of particle trajectory lengths with the zenith angle distribution expected for the incoherent muon background;

3. Affordable and scalable sensor network concept named CREDO-Maze has been presented in Ref. [13, 14]: small scintillator based detectors might form a portable network which is capable of detecting air showers (potentially components of CRE), and which can be scaled up by inviting schools and individual science enthusiasts that are interested in both cosmic ray based education and in contributing directly to scientific research;

4. Detector simulations and studies that allow proper interpretation of the signal received by a network of small sensors are presented in Refs. [15, 16]; 

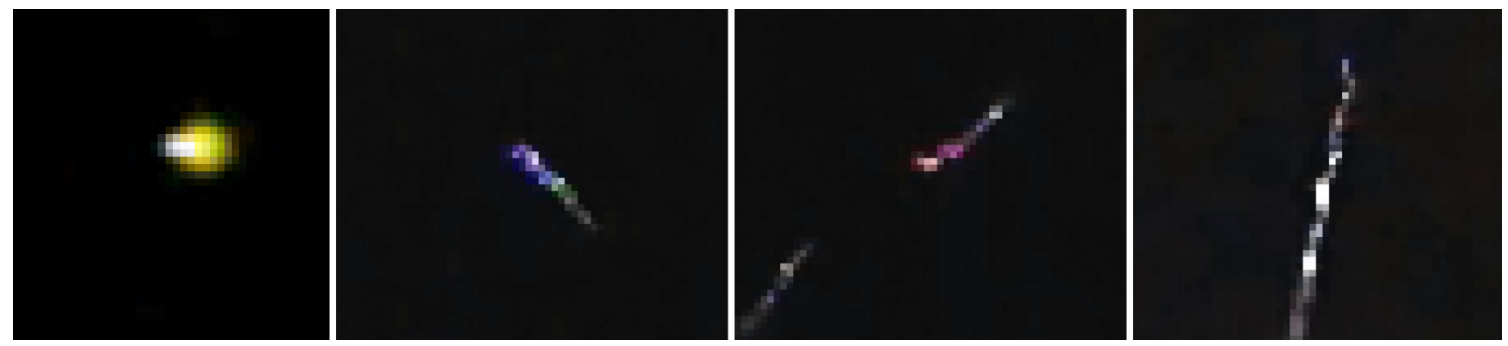

Figure 2: The variety of particle tracks candidates as registered by a smartphone using the CREDO Detector app.

5. A method to recognize two air showers as CRE components has been proposed in Ref. [17];

6. Societal aspects of the CREDO program [18]: The specific collection of science objectives related to a program dedicated to CRE which involves a global network of affordable detectors requires a necessary work in the direction of science communication, popularization, outreach, education and other activities related to public engagement and citizen science. These activities and a perspective of future plans are summarized in Ref. [18].

To date, most of the data collected by CREDO comes from smartphones with the CREDO Detector app, operating on the Android system with already more than 10.5 million detections, and with the Cosmic Ray App dedicated to iOS devices with more than 7 million detections. An example set of particle track candidates collected by CREDO Detector is presented in Fig. 2. Another important example of the infrastructure working within the CREDO Collaboration, although not yet connected to the central system, is HEAMS (High Energy Astrophysics Muon System): an array of muon detectors operated by the University of Adelaide, Australia, consisting of several one square meter scintillator muon detectors in two locations distant by $40 \mathrm{~km}$. These two example resources, and the corresponding studies, in particular the one demonstrating the feasibility of identification of muons with smartphones [11, 12], illustrate the main concept and potential of a global network of affordable radiation sensors. Correspondingly, the already achieved interest of the public, including in particular many young science enthusiasts and their teachers, promises a sustainable growth of the network and a continuous support for all the scientific projects to be carried out using the CREDO resources.

\section{The first observational encouragements and the multi-messenger/multi-mission potential}

Among the first interesting experimental results obtained within the CREDO program we list:

1. The search for bursts of air showers of primary energies of $0.1 \mathrm{PeV}$ using a small air shower array [8]: if air shower events form clusters with arrival times of individual events spread over a considerably wide period of time, e.g. on the scale of seconds or minutes as might have been indicated in other experiments (see the discussion and the references in Refs. [8]), then such an observation might require shifting the astroparticle physics paradigm. In Ref. [8] we 
present five-fold cluster candidates in which air shower arrive within 40 seconds with similar regions of the sky.

2. The search for correlations between the secondary air shower cosmic rays and global number of earthquakes: the study being prepared for publication promises opening a new perspective of a contribution from the cosmic ray field to the multi-messenger, interdisciplinary earthquake early warning system being considered by both the astroparticle physics and geophysics communities.

The listed results point to the possibility of observing CRE being just around the corner, and also to an additional "side-effect" realm of inter- and transdisciplinary application of a global network of radiation sensors, as it is being proposed and organized by the CREDO Collaboration. And once CRE are observed, we would open a new information channel to observe the Universe through, directly supporting an complementing all the multi-messenger studies in the field of astroparticle physics. Moreover, once a global network of radiation sensors is optimally organized and operational, it must bring a variety of multi-mission research opportunities and synergies, having the aforementioned perspective of a contribution to an early warning system against earthquakes as a flagship example. We have been excited about the upcoming future and eager to welcome more collaborators to make this future scientifically even more brighter then we expect.

\section{Summary and Outlook}

Once CRE are observed, a new observation channel of the Universe will be open, and the information from this channel can point us back to the interactions at energies close to the GUT scale, leading possibly to a breakthrough in physics. On the other hand, if CRE are not observed, we will set the new upper limits constraining selected theories. Moreover, since the CREDO concept and strategy offers inter- and transdisciplinary opportunities, the advances of the studies proposed here will automatically contribute to many external studies and scientific achievements as well. Feel invited!

\section{Acknowledgements}

We acknowledge the leading role in the CREDO Collaboration and the commitments to this research made by the Institute of Nuclear Physics Polish Academy of Science. This research has been supported in part also by PLGrid Infrastructure and we warmly thank the staff at ACC Cyfronet AGH-UST for their always helpful supercomputing support.

\section{References}

[1] P. Homola, et al. for the CREDO Collaboration, Cosmic Ray Extremely Distributed Observatory, Symmetry 12, no. 11, (2020) 1835. DOI: 10.3390/sym12111835

[2] K. Almeida Cheminant, D. Góra, et al. for the CREDO Collaboration, Event rates of UHE photons cascading in the geomagnetic field at CTA-North, in proceedings of the $37^{\text {th }}$ ICRC 2021, PoS (ICRC2021) 726 
[3] K. Almeida Cheminant, et al. for the CREDO Collaboration, Search for ultra-high energy photons through preshower effect with gamma-ray telescopes: Study of CTA-North efficiency, Astropart. Phys. 123, (2020) 102489. DOI : 10.1016/j . astropartphys. 2020.102489

[4] D. Alvarez-Castillo, et al. for the CREDO Collaboration, Simulations of Cosmic Ray Ensembles originated nearby the Sun, in proceedings of the $37^{\text {th }}$ ICRC 2021, PoS (ICRC2021) 457

[5] O. Sushchov, et al. for the CREDO Collaboration, Formation and propagation of cosmic-ray ensembles, in proceedings of the $37^{\text {th }}$ ICRC 2021, PoS (ICRC2021) 465

[6] A. Tursunov, et al. for the CREDO Collaboration, Acceleration of UHECR by local supermassive black hole candidates, in proceedings of the $37^{\text {th }}$ ICRC 2021, PoS (ICRC2021) 471

[7] A. Tursunov, et al. for the CREDO Collaboration, Probing UHECR and cosmic ray ensemble scenarios with a global CREDO network, in proceedings of the $37^{\text {th }}$ ICRC 2021, PoS (ICRC2021) 472

[8] R. Clay, J. Singh, et al. for the CREDO Collaboration, A search for bursts at 0.1 PeV with a small air shower array, in proceedings of the $37^{\text {th }}$ ICRC 2021, PoS (ICRC2021) 298

[9] $\mathrm{L}$. Bibrzycki, et al. for the CREDO Collaboration, Machine learning aided noise filtration and signal classification of the CREDO smartphone data, in proceedings of the $37^{\text {th }}$ ICRC 2021, PoS (ICRC2021) 227

[10] Ł. Bibrzycki, et al. for the CREDO Collaboration, Towards A Global Cosmic Ray Sensor Network: CREDO Detector as the First Open-Source Mobile Application Enabling Detection of Penetrating Radiation, Symmetry 12, no. 11, (2020) 1802. DOI: 10.3390/sym 12111802

[11] T. Wibig, et al. for the CREDO Collaboration, Determination of Zenith Angle Dependence of Incoherent Cosmic Ray Muon Flux Using Smartphones of the CREDO Project, in proceedings of the $37^{\text {th }}$ ICRC 2021, PoS (ICRC2021) 199

[12] T. Wibig, et al. for the CREDO Collaboration, Determination of Zenith Angle Dependence of Incoherent Cosmic Ray Muon Flux Using Smartphones of the CREDO Project, Appl. Sci. 11, (2021) 1185. DOI : 10.3390/app11031185

[13] M. Karbowiak, et al. for the CREDO Collaboration, Small shower array for education purposes. CREDO-Maze Project, in proceedings of the $37^{\text {th }}$ ICRC 2021, PoS (ICRC2021) 219

[14] M. Karbowiak, T. Wibig, et al. for the CREDO Collaboration, The first CREDO registration of extensive air shower, Phys. Edu. 55, no. 5, (2020) 055021. DOI : $10.1088 / 1361-6552 / \mathrm{ab} 9 \mathrm{dbc}$

[15] J. Pryga, et al. for the CREDO Collaboration, Analysis of capability of detection of extensive air showers by simple scintillator detectors, in proceedings of the $37^{\text {th }}$ ICRC 2021, PoS (ICRC2021) 430

[16] W. Stanek, et al. for the CREDO Collaboration, Particle density fluctuations and correlations in low energy Cosmic-Ray showers simulated with CORSIKA, in proceedings of the $37^{\text {th }}$ ICRC 2021, PoS (ICRC2021) 462

[17] Yu. Verbetsky, M. Svanidze, D. Beznosko, P. Homola, et al. for the CREDO Collaboration, On the possible method of identification of two probably cognate Extensive Air Showers, in proceedings of the $37^{\text {th }}$ ICRC 2021, PoS (ICRC2021) 424

[18] R. Kamiński, J. Firla, et al. for the CREDO Collaboration, Cosmic rays and the structure of the universe studied in Cosmic Ray Extremely Distributed Observatory with citizen science, in proceedings of the $37^{\text {th }}$ ICRC 2021, PoS (ICRC2021) 1370 


\section{Full Authors List: Cosmic Ray Extremely Distributed Observatory}

Piotr Homola ${ }^{1}$, David E. Alvarez Castillo ${ }^{1,2}$, Kevin Almeida Cheminant ${ }^{1}$, Dmitriy Beznosko ${ }^{3}$, Nikolai Budnev ${ }^{4}$, Dariusz Góra ${ }^{1}$, Alok C. Gupta $^{5}$, Bohdan Hnatyk ${ }^{6}$, Marcin Kasztelan ${ }^{7}$, Peter Kovacs ${ }^{8}$, Bartosz Lozowski ${ }^{9}$, Mikhail V. Medvedev ${ }^{10,11}$, Justyna Miszczyk ${ }^{1}$, Alona Mozgova $^{6}$, Vahab Nazari ${ }^{2,1}$, Michał Niedźwiecki ${ }^{12}$, Maciej Pawlik ${ }^{13,14}$, Matías Rosas ${ }^{15}$, Krzysztof Rzecki ${ }^{14}$, Katarzyna Smelcerz ${ }^{12}$, Karel Smolek ${ }^{16}$, Jarosław Stasielak ${ }^{1}$, Sławomir Stuglik ${ }^{1}$, Oleksandr Sushchov ${ }^{1}$, Manana Svanidze ${ }^{17}$, Arman Tursunov ${ }^{18}$, Jose M. Vaquero ${ }^{19}$ Yuri Verbetsky ${ }^{17}$, Tadeusz Wibig ${ }^{20}$, Krzysztof Woźniak ${ }^{1}$ Jilberto Zamora-Saa ${ }^{21}$.

${ }^{1}$ Institute of Nuclear Physics Polish Academy of Sciences, Radzikowskiego 152, 31-342 Kraków, Poland. ${ }^{2}$ Joint Institute for Nuclear Research, Dubna, 141980 Russia. ${ }^{3}$ Clayton State University, Morrow, Georgia, USA. ${ }^{4}$ Irkutsk State University, Russia. ${ }^{5}$ Aryabhatta Research Institue of Observational Sciences (ARIES), Manora Peak, Nainital 263001, India. ${ }^{6}$ Astronomical Observatory of Taras Shevchenko National University of Kyiv, 04053 Kyiv, Ukraine. ${ }^{7}$ National Centre for Nuclear Research, Andrzeja Soltana 7, 05-400 Otwock-Świerk, Poland. ${ }^{8}$ Institute for Particle and Nuclear Physics, Wigner Research Centre for Physics, 1121 Budapest, KonkolyThege Miklós út 29-33, Hungary. ${ }^{9}$ Faculty of Natural Sciences, University of Silesia in Katowice, Bankowa 9, 40-007 Katowice, Poland. ${ }^{10}$ Department of Physics and Astronomy, University of Kansas, Lawrence, KS 66045, USA. ${ }^{11}$ Laboratory for Nuclear Science, Massachusetts Institute of Technology, Cambridge, MA 02139, USA. ${ }^{12}$ Department of Computer Science, Faculty of Computer Science and Telecommunications, Cracow University of Technology, Warszawska 24, 31-155 Kraków, Poland. ${ }^{13}$ ACC Cyfronet AGH-UST, 30-950 Kraków, Poland. ${ }^{14}$ AGH University of Science and Technology, Mickiewicz Ave., 30-059 Kraków, Poland. ${ }^{15}$ Liceo 6 Francisco Bauzá, Montevideo, Uruguay. ${ }^{16}$ Institute of Experimental and Applied Physics, Czech Technical University in Prague. ${ }^{17} \mathrm{E}$. Andronikashvili Institute of Physics under Tbilisi State University, Georgia. ${ }^{18}$ Research Centre for Theoretical Physics and Astrophysics, Institute of Physics, Silesian University in Opava, Bezručovo nám. 13, CZ-74601 Opava, Czech Republic. ${ }^{19}$ Department of Physics, University of Extremadura, 06800 Mérida, Spain. ${ }^{20}$ University of Łódź, Faculty of Physics and Applied Informatics, 90-236 Łódź, Pomorska 149/153, Poland. ${ }^{21}$ Universidad Andres Bello, Departamento de Ciencias Fisicas, Facultad de Ciencias Exactas, Avenida Republica 498, Santiago, Chile. 